\title{
LOS CUMÁCEOS (CRUSTACEA: PERACARIDA) DEL ARCHIPIÉLAGO CUBANO
}

\author{
Manuel Ortiz y Rogelio Lalana ${ }^{2}$ \\ ${ }^{1}$ Laboratorio de crustáceos, Facultad de Estudios Superiores Iztacala, UNAM, México. \\ ortiztouzet@yahoo.com. ${ }^{2}$ Centro de Investigaciones Marinas, \\ Universidad de La Habana, Cuba. rlalana@cim.uh.cu.
}

\section{RESUMEN}

Se presenta un recuento histórico sobre las investigaciones previas desarrolladas sobre los cumáceos cubanos. Además, se ofrecen los caracteres diagnósticos para reconocer las cuatro familias representadas en Cuba: Bodotriidae (Cyclaspis y Vaunthompsonia); Diastylidae (Leptostylis y Oxyurostylis); Leuconidae (Leucon) y Nannastacidae (Campylaspis, Cubanocuma, Cumella y Elassocumella). Un total de 27 especies registradas para el país podrán ser clasificadas mediante las claves dicotómicas que se ofrecen en este trabajo. También se presenta una diagnosis para cada familia y género, así como una figura para cada especie.

Palabras clave: Cumacea, claves de identificación, especies cubanas.

\section{THE CUMACEAN SHRIMPS (CRUSTACEA: PERACARIDA) FROM THE CUBAN ARCHIPELAGO}

\begin{abstract}
A brief history review regarding the previous research on the study of the Cuban cumacean is presented. The diagnostic characters to recognize the four families recorded in Cuba: Bodotriidae (Cyclaspis and Vaunthompsonia); Diastylidae (Leptostylis and Oxyurostylis); Leuconidae (Leucon) and Nannastacidae (Campylaspis, Cubanocuma, Cumella and Elassocumella), are given. A total of 27 species recorded for the country can be classified by using the given dichotomy keys. The diagnosis for each family and genus, as well as a figure for each species, are also pointed out.
\end{abstract}

Keywords: Cumacea, identification keys, Cuban species.

\section{INTRODUCCIÓN}

Los cumáceos, más conocidos como "camarones encapuchados", constituyen un orden de peracáridos marinos o estuarinos que han resultado muy poco estudiados en las aguas cubanas. Su tamaño suele oscilar entre uno y $35 \mathrm{~mm}$. Viven generalmente semienterrados en el sedimento blando, con su región anterior ligeramente erguida sobre la superficie del sustrato. También son localizados bajo las rocas, entre macroalgas y fanerógamas (Heard et al., 2007; Petrescu, 2002, 2004).

Es un grupo conocido desde hace tiempo, aunque su taxonomía no estuvo bien definida por poseer un marcado dimorfismo sexual, hasta que se fueron reclasificando correctamente muchas especies que habían sido descritas como nuevas, resultando ser algunas el sexo opuesto de otra también descrita (Calman, 1907, 1912). Aún así, resulta a veces muy difícil determinar en una muestra de sedimento, ante varios especímenes del grupo, cuándo las hembras y los machos corresponden a una misma especie. 
Morfología. Es un grupo de peracáridos muy fácil de reconocer, pues su cuerpo posee un carapacho o cefalotórax abultado, que generalmente lleva un rostro anterior, protegido a cada lado por dos estructuras únicas entre los crustáceos, denominadas pseudorostros. Tanto en el rostro como en el pseudorostro pueden llevar varios ojos, uno o ninguno. Pueden presentar un sifón anterior en ciertos casos. Poseen un par de antenas, a veces tan largas en los machos, que pueden llegar hasta el final telson. En las hembras son muy cortas. Su carapacho deja sin cubrir los últimos tres segmentos del pereón (figs. 1 y 2). Le sigue un abdomen alargado y estrecho, después el telson y los urópodos. Los machos casi siempre llevan pleópodos en todos o algunos de los segmentos abdominales, mientras que faltan en las hembras. Ciertas especies poseen un par de sifones anales, que surgen al final del telson.

Sus piezas bucales, a diferencia de los restantes peracáridos, consisten en tres pares de maxilípedos, dispuestos muy separados entre sí, dos pares de maxilas y un par de mandíbulas. En el pereón llevan cinco pares de pereópodos; los pleópodos generalmente están presentes en los machos, sin embargo, casi siempre faltan en las hembras (figs. 1-3).
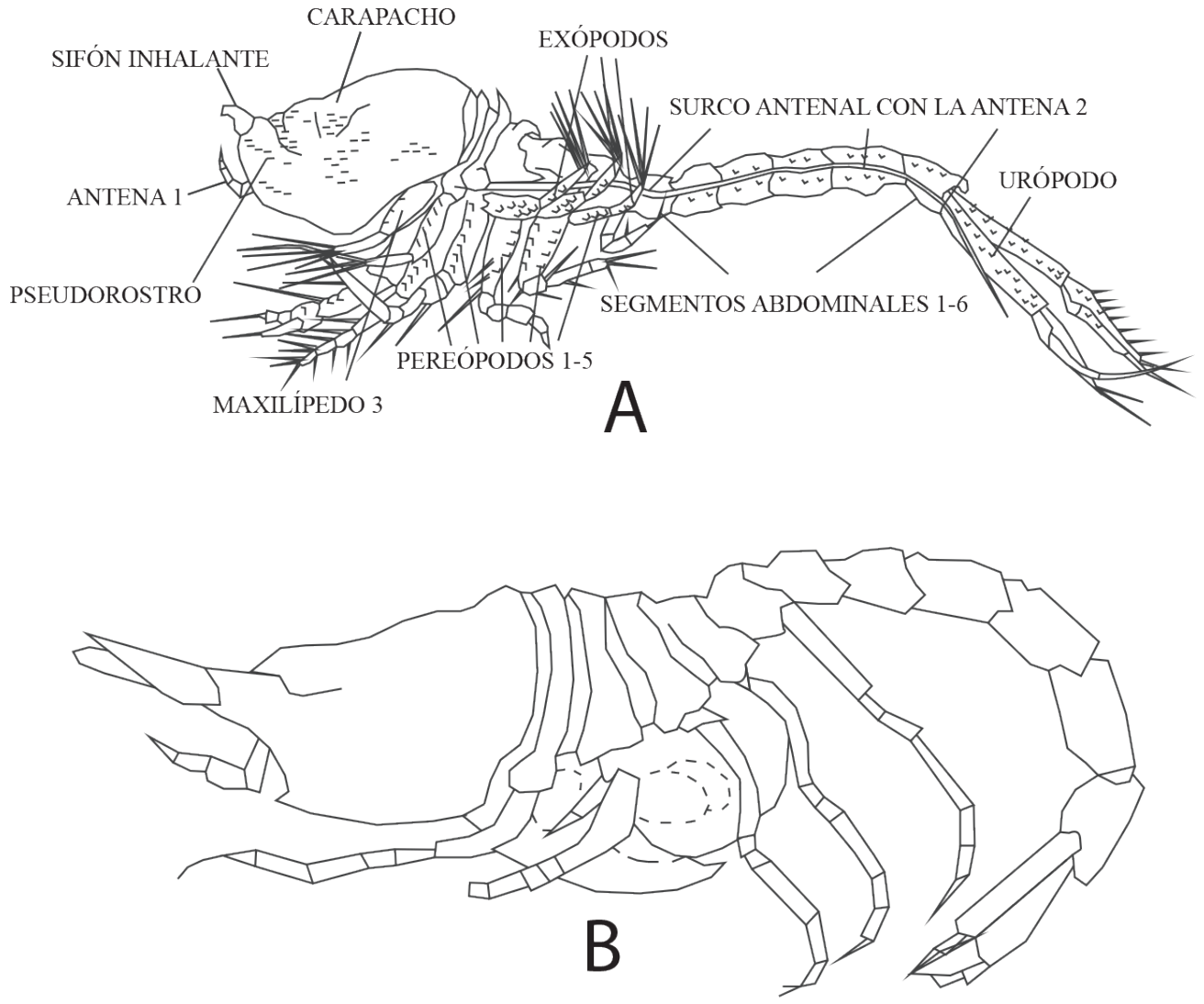

FIGURA 1. A-B. Vista lateral mostrando la morfología externa de los cumáceos. A, macho. B, hembra ovígera, con huevos en su marsupio. 


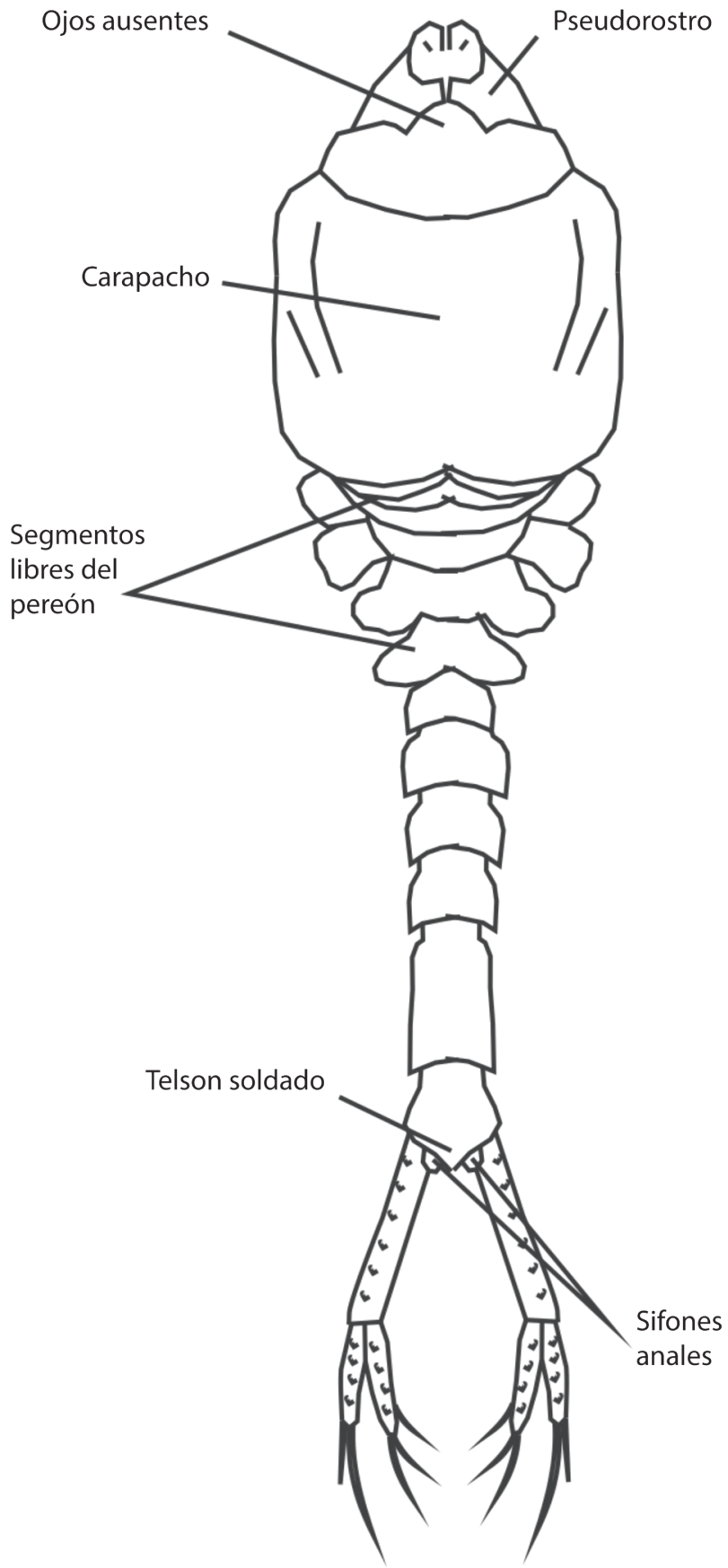

FIGURA2. Vista dorsal de un cumáceo mostrando sus detalles morfológicos. 


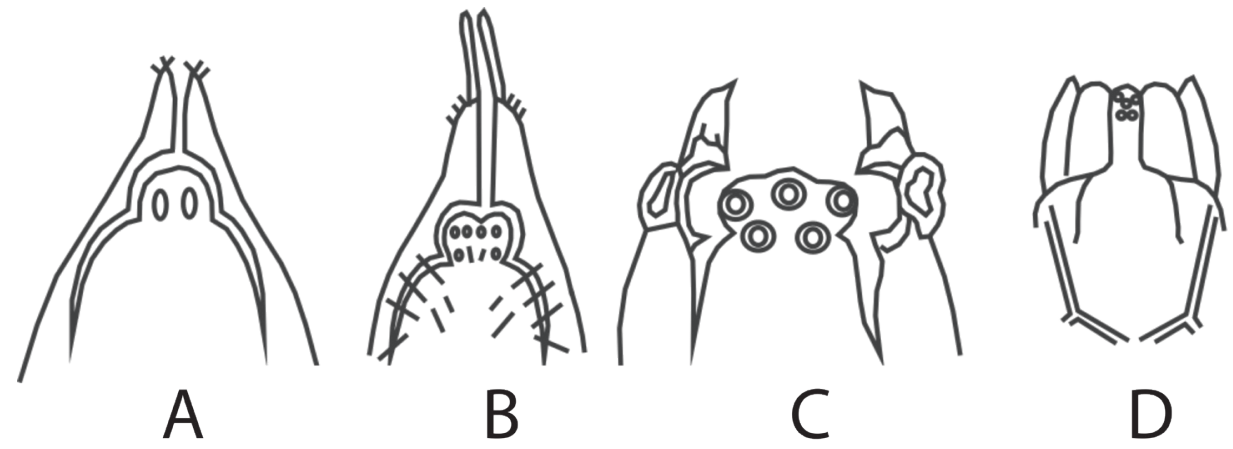

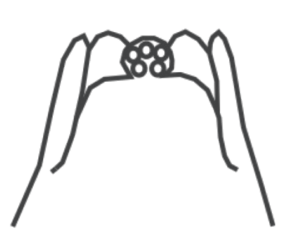

E

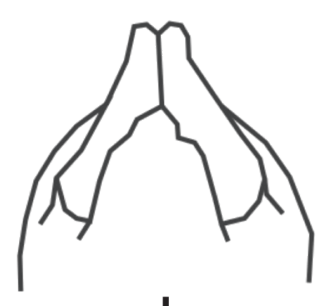

।

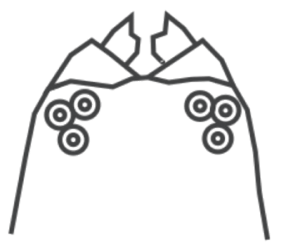

$\mathrm{M}$

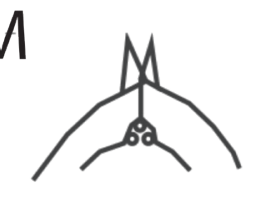

$\mathrm{Q}$

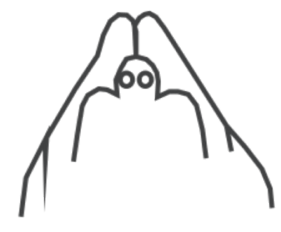

$\mathrm{F}$
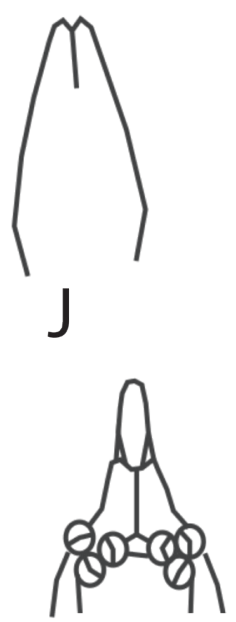

$\mathrm{N}$

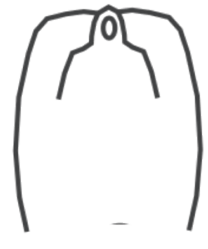

G

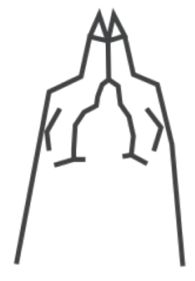

K

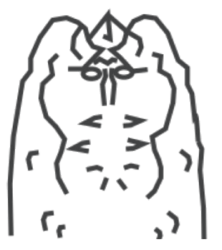

0

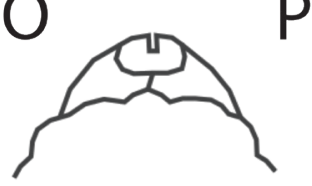

$\mathrm{R}$

FIGURA 3. A-R. Vista dorsal de la región anterior del carapacho de los géneros. A, B, C, Cumella; D, E, F, G, H, Cyclaspis; I, J, Oxyurostylis; K, Eudorella; L, Vaunthompsonia; M, N, Nannastacus; O, Cubanocuma; P, Diastylis; Q, R, Campylaspis, Eudorella, Nannastacus y Diastylis. 
En estos peracáridos, los maxilípedos están dispuestos ventralmente, bien separados entre sí (fig. 4A). Casi siempre el primero de ellos es respiratorio, pudiendo llevar pliegues en su parte basal y un sifón respiratorio distalmente (fig. 4B), otros son más simples (figs. 4C, 4D). Tanto sus maxilípedos como sus pereópodos pueden presentar o carecer de exopoditos. Los pleópodos que aparecen únicamente en los machos y poseen exo y endopoditos simples o con el endopodito formando un lóbulo esternal que protege al exopodito correspondiente (fig. 5).

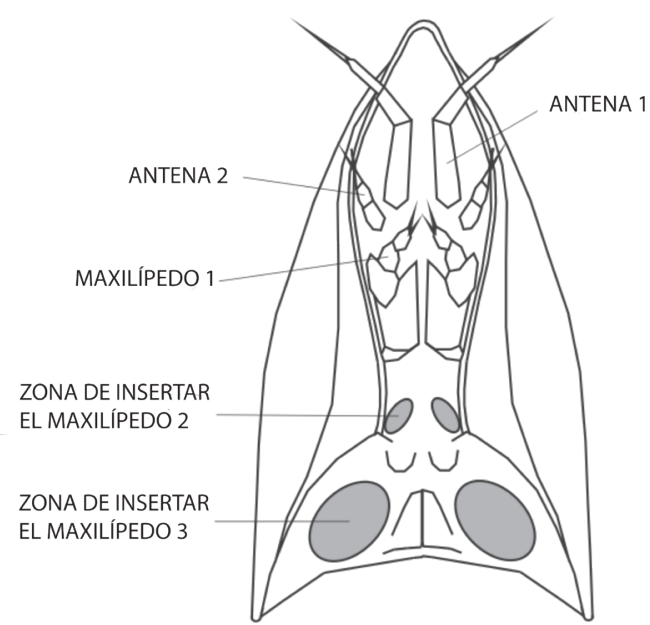

A
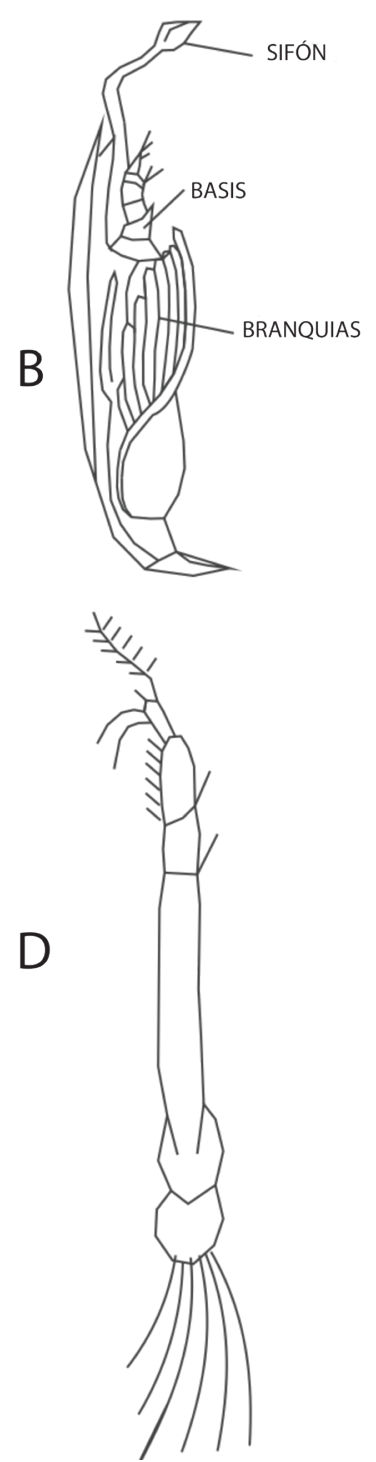

FIGURA 4. A, carapacho en vista ventral para mostrar los sitios de inserción de los maxilípedos. $\mathrm{B}$, maxilípedo respiratorio. C, maxilípedo típico de Campylaspis. D, maxilípedo típico de los bodótridos. 


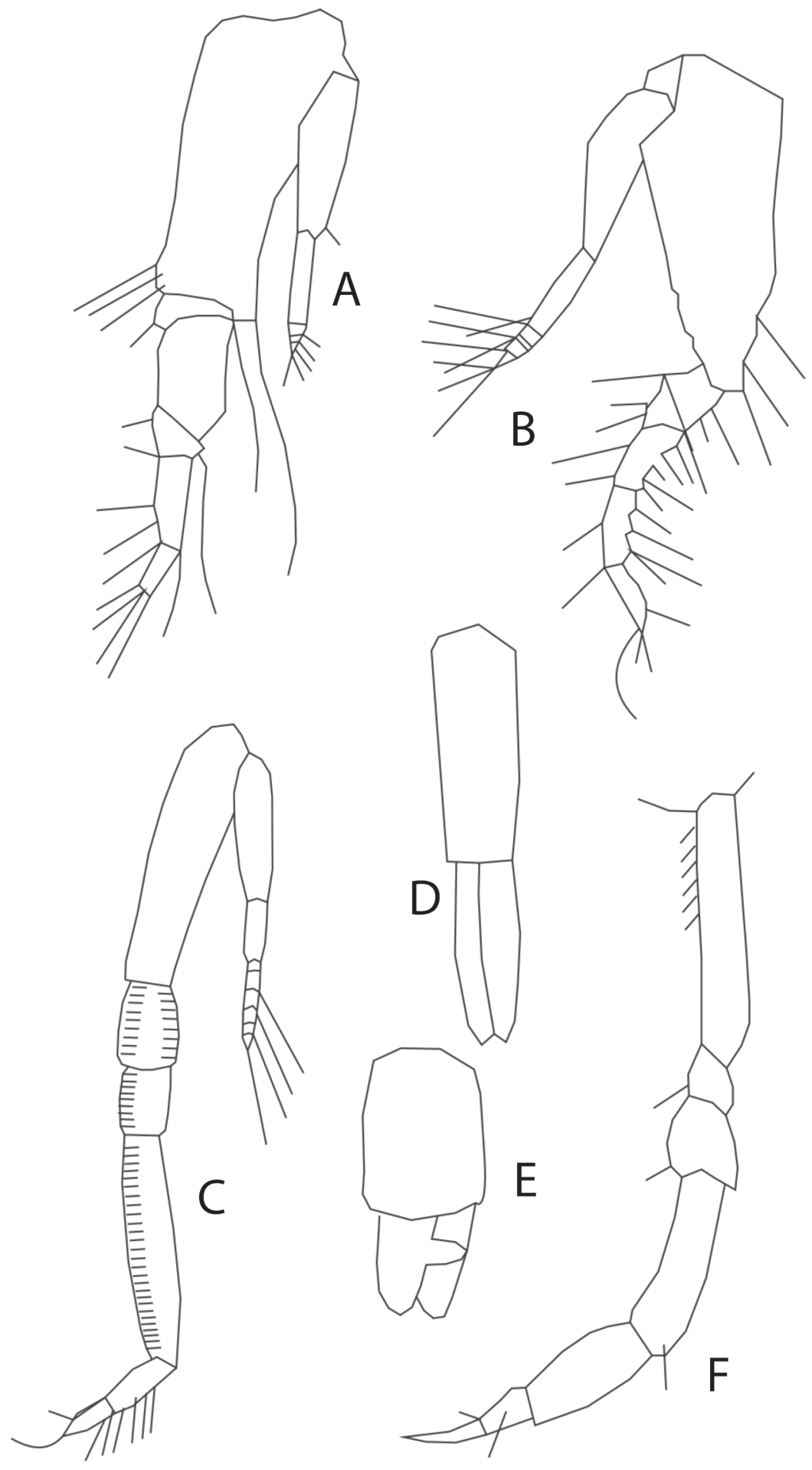

FIGURA 5. A-C, tipos de apéndices con exopoditos más comunes en los cumáceos. D, pleópodo normal. E, pleópodo con endópodo formando proceso external, propio de los bodótridos. F, apéndice simple. 


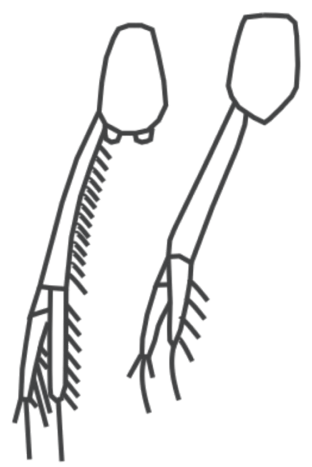

A

B

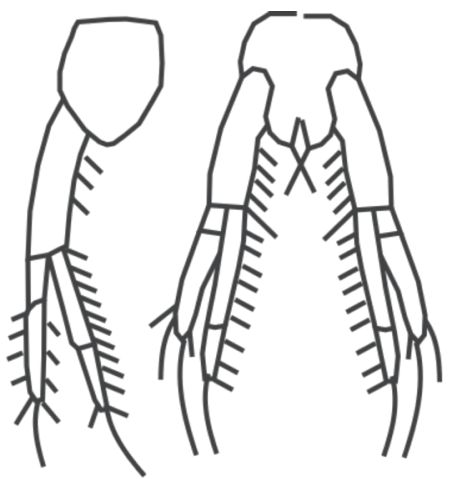

C

D

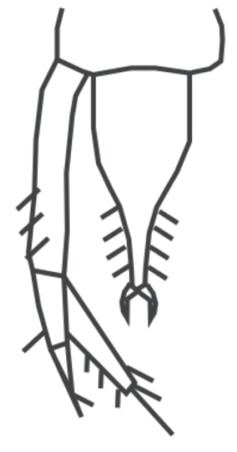

$\mathrm{E}$

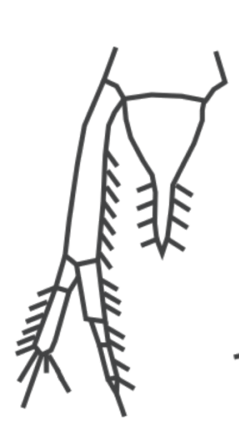

$\mathrm{F}$

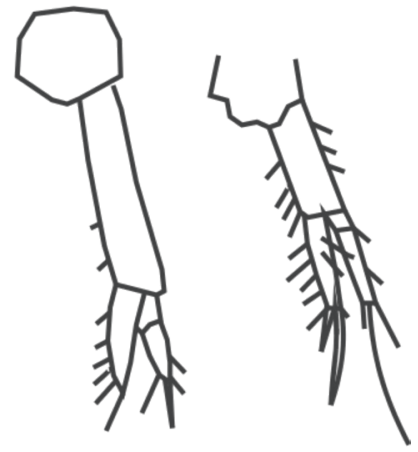

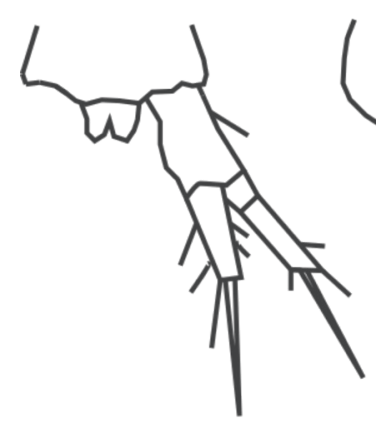

$\mathrm{K}$
G

$\mathrm{H}$

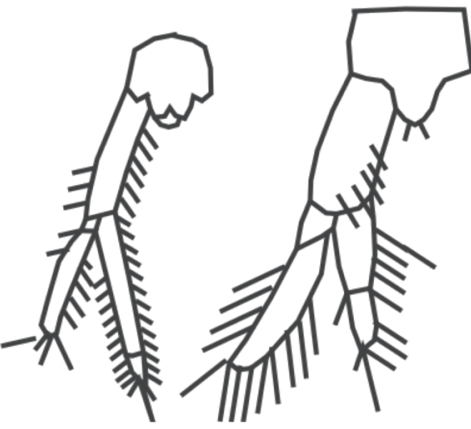

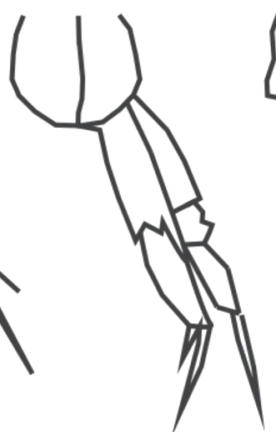

$\mathrm{L}$

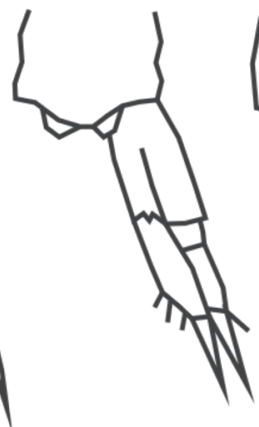

M

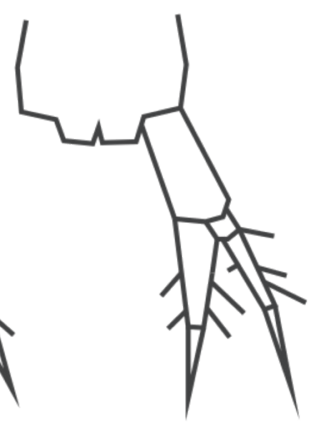

$\mathrm{N}$

FIGURA 6. A-N. Urópodos más telsones. A, B, Cyclaspis. C, D, Vaunthompsonia. E, Diastylis. F, Oxyurostylis. G, H, Leucon. I, J, Campylaspis. K, Cubanocuma. L-N, Cumella. 
El telson puede aparecer libre o soldado al sexto segmento abdominal. Los urópodos varían en cuanto al número de artejos. En ciertos casos, estos animales desarrollan sifones anales (figs. 2 y 6).

Las piezas bucales, el número de exopoditos presentes en los pereópodos, la morfología del telson, si está libre o soldado, los ornamentos del carapacho, los artejos y las setas o setas robustas de los urópodos, son todos de un gran interés diagnóstico. Se trata de los únicos peracáridos que presentan generalmente la base del primero o segundo par de maxilípedos modificados para la respiración.

Métodos de colecta. Pueden ser colectados con rastras usadas para organismos bentónicos pequeños, con trampas de luz, con lavados de pedacería de coral o durante la noche, desde una embarcación, con una luz colocada cerca de la superficie del mar, con el auxilio de una red manual (para los machos que poseen pleópodos y nadan).

Historia. La primera especie citada para Cuba fue Cumella vicina Zimmer, 1943 (Petrescu, 2004). Casi tres décadas después, Băcescu, 1971, describe el nanastásido Cumella aglutinanta, actualmente Schizotrema aglutinanta (Băcescu, 1971). Unos años más tarde, Băcescu y Muradian (1977a; 1977b), describen el género Cubanocuma y las especies C. gutzui y Cumella gomoiui. Después, las especies Cyclaspis bacescui Omholt y Heard, 1983 y C. goesii Sars, 1871, son citadas para Cuba por Băcescu y Ortiz (1984).

Por otra parte, Ortiz y Lalana $(1988 ; 1994)$ citan otros cumáceos para Cuba: Cyclaspis băcescui, C. longipes, Vaunthompsonia floridanas y V. minor (Iphinoe sp., no debe considerarse, por ser una especie típica del Mar Medeterráneo). Además, Ortiz y Lalana (1998), publican la lista de crustáceos no decápodos de Cuba, donde se citan otras 11 especies de cumáceos. Unos años antes, Donath-Hernández (1988), cita a Cyclaspis unicornis para las aguas cubanas. Además, C. goesi (Sars, 1871) y Cumella unicornis Calman, 1907, son citadas por Petrescu (2002). En ese mismo año, es descrito el bodótrido Cyclaspis iorgui Ortiz y Lalana, 2002.

Dos años después, Varela et al. (2003), mencionan a los nanastácidos Cumella micruropus, actualmente Elassocumella micruropus (Zimmer, 1861) y C. serrata para la península de Guanahacabibes, en la zona más occidental de la isla de Cuba. Posteriormente, Lalana et al. (2005) citan nuevamente a E. micruropus y adicionan el diastílido Oxyurostylus cf. smithi, para el país.

Finalmente, Ortiz y Lalana (1998), presentan la lista de los crustáceos no decápodos cubanos, donde se citan 11 de las especies citadas con anterioridad. Además, Heard et al. (2007) citan varias especies para Cuba, al publicar una guía para la identificación de los cumáceos de la Florida y regiones adyacentes. Las claves cubanas para identificar crustáceos de Ortiz y Lalana (2010), ofrecen cómo determinar las familias de los cumáceos cubanos.

En Cuba, el estudio de estos peracáridos permanece muy deficiente, como denota la breve historia antes presentada. Estudios diversos en zonas cercanas del Golfo y el Mar Caribe, registran un número muchísimo mayor de especies (Băcescu, 1971: Băcescu y Muradian, 1977; Donath Hernández, 1988; Heard et al., 2007; Watling, 1991; Petrescu, 1996, 2002). El trabajo más importante sobre cumáceos cubanos es el de Petrescu (2004). Se basa en una colección realizada por Gutu Modest, al llevarse a cabo una expedición conjunta organizada en los años 70 por el Museo Nacional de Historia Natural "Grigore Antipa" de Bucarest, Rumania y el Instituto de Oceanología, de la Academia de Ciencias de Cuba, el que se desarrolló en las aguas de la región suroriental cubana. 
Hasta el presente se han registrado cuatro familias, 11 géneros y 29 especies para el archipiélago cubano. Los géneros mejor representados son Cumella, con 11 especies y Cyclaspis, con seis.

\section{OBJETIVO}

-Actualizar el estado del conocimiento de los cumáceos cubanos y contribuir a su identificación correcta, mediante claves y figuras apropiadas.

\section{MATERIALES Y MÉTODOS}

Los táxones que se presentan a continuación han sido citados previamente para las aguas someras del archipiélago cubano por diversos autores.

Los cumáceos presentan un dimorfismo sexual muy marcado, por lo cual se ha decidido presentar las claves de identificación para las hembras, las que generalmente son más abundantes que los machos. De estos últimos, en los casos pertinentes, se ofrecen datos complementarios.

Las claves que se ofrecen se han confeccionado tomando en cuenta, fundamentalmente, caracteres externos de fácil visualización. Los diferentes tipos de carapachos en vista dorsal se presentan en la figura 3, mientras en la figura 6 se muestran los télsones y urópodos de los géneros Campylaspis, Diastylis, Eudorella y Leucon, porque, según su distribución en la región, podrán aparecer en futuras colectas en las aguas marinas del país.

Las figuras se han confeccionado con el programa Corel Draw X4. Muchas se basan en las presentadas en los trabajos de Băcescu (1971, 1977a), Petrescu (1996, 2002), Ortiz y Lalana (2002) y Heard et al. (2007). Todas son de hembras adultas. La validez de las especies citadas en este trabajo ha sido verificada de acuerdo con WoRMS (2017).

\section{RESULTADOS}

\section{Familia BODOTRIIDAE Scott, 1901}

La integran a nivel mundial cerca de 35 géneros y más de 300 especies (Heard et al., 2007). Presentan el carapacho ovoidal; rostro y pseudorostros poco prominentes; sin sifones respiratorios; machos y hembras del mismo tamaño (la subfamilia Mancocumatinae, no presente en Cuba, se distingue por presentar machos más pequeños que las hembras); machos de antenas con flagelo muy largo; cinco pares de pleópodos; pleópodos con el endópodo formando un proceso externo que protege al exópodo; exópodo presente en el tercer maxilípedo y en el primer par de pereópodos; en los restantes pereópodos su número es variable, en ambos sexos, donde machos y hembras son del mismo tamaño); maxilípedo dos del tipo "Bodotrido" (fig. 4D); endopodito del urópodo con uno o dos artejos. Con el telson muy reducido y soldado con el sexto segmento pleonal. En el género Spilocuma, no citado aún para Cuba, los machos no llevan pleópodos. Con dos subfamilias: Bodotriinae y Vaunthompsoniinae. 
Subfamilia BODOTRIINAE Scott, 1901

Diagnosis. Exópodos presentes solamente en el maxilípedo tres y en el primer par de pereópodos, en ambos sexos; endopodito del urópodo con un solo artejo.

Género Cyclaspis Sars, 1865

Diagnosis. Machos con antenas nunca más largas que el carapacho; con exópodos presentes en el maxilípedo 3 y pereiópodo 1, en ambos sexos; hembras con uno o dos artejos en la antena.

Especies citadas para Cuba: C. bacescui Omholt y Heard, 1983; C. goesi (Sars, 1871); C. iorgui Ortizy Lalana, 2002; C. longipes Calman, 1907; C. simonae Petrescu, Iliffe y Sarbu, 1993; C. unicornis Calman, 1907.

\section{CLAVE PARA LAS HEMBRAS DE LAS ESPECIES CUBANAS}

1A. Carapacho en vista lateral totalmente liso; sin dientes mediodorsales; primer par de pereópodos tan delgados como los restantes ------- Cyclaspis longipes (fig. 7A).

1B. Carapacho con dientes medio dorsales, estrías o crestas laterales; primer par de pereópodos más ancho que los restantes $-2$

2A. Carapacho con un diente medio dorsal en el tercio anterior del carapacho; resto del carapacho liso $-3$

2B. Carapacho sin el mencionado diente; resto del carapacho con ornamentos 4

3A. Pleón con crestas laterales longitudinales; sexto segmento pleonal subrectandular en vista dorsal, más largo que ancho, con una cresta longitudinal discreta, en la línea media Cyclaspis goesi (fig. 7B).

3B. Pleón con crestas finas laterales longitudinales; sexto segmento pleonal subpentagonal en vista dorsal, con la cresta longitudinal discreta ausente

$$
\text { Cyclaspis unicornis (fig. 8A). }
$$

4A. Carapacho con cresta dorsal longitudinal doble; con 11 estrías finas algo oblicuas sobre los laterales del carapacho Cyclaspis simonae (fig. 8B).

4B. Carapacho sin cresta dorsal longitudinal doble; con más de 11 estrías finas oblicuas laterales $-5$

5A. Carapacho con pseudorostro corto en vista lateral (un tercio o menos del largo del carapacho); con algunas estrías anteriormente bifurcadas; rostro en vista dorsal, con una estrangulación posterior a 9 lentes dispuestos muy juntos, formando un arco -Cyclaspis bacescui (fig. 9A).

5B. Carapacho con pseudorostro largo (más de un tercio del largo del carapacho en vista lateral); con 14 estrías sin bifurcase anteriormente; rostro en vista dorsal, lingüiforme no estrangulado con 7 lentes bien separados entre sí 


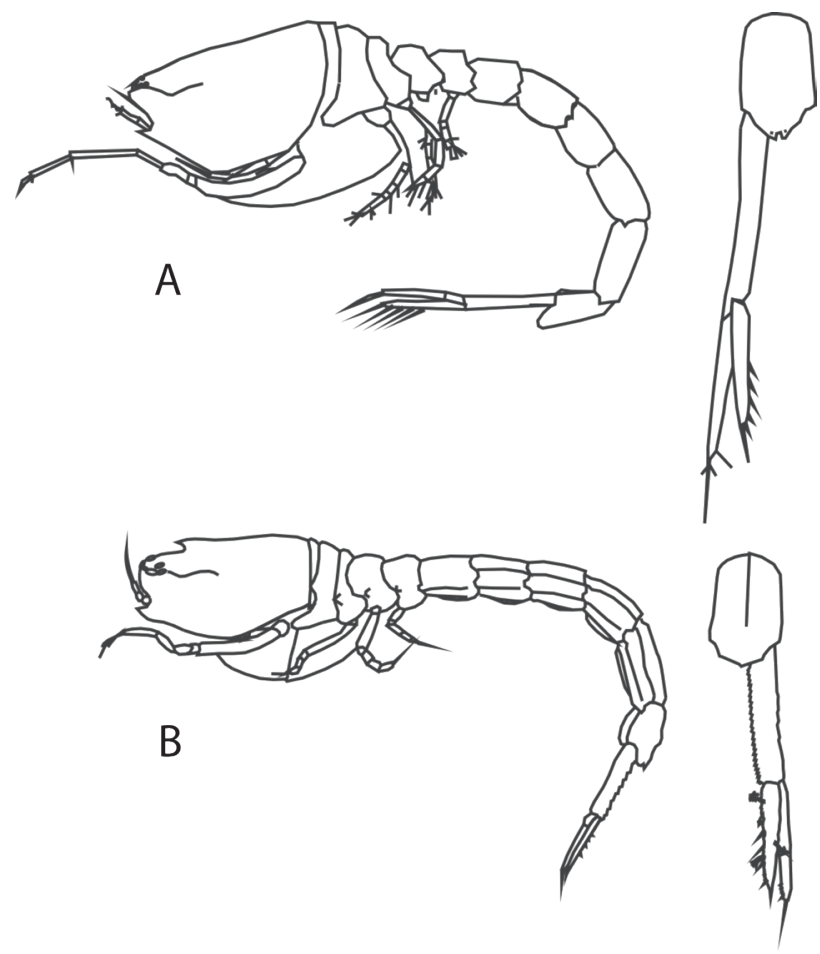

FIGURA 7. A-B. Vista lateral del cuerpo, telson y urópodos. A, Cyclaspis longipes +. B, C. goesi +.
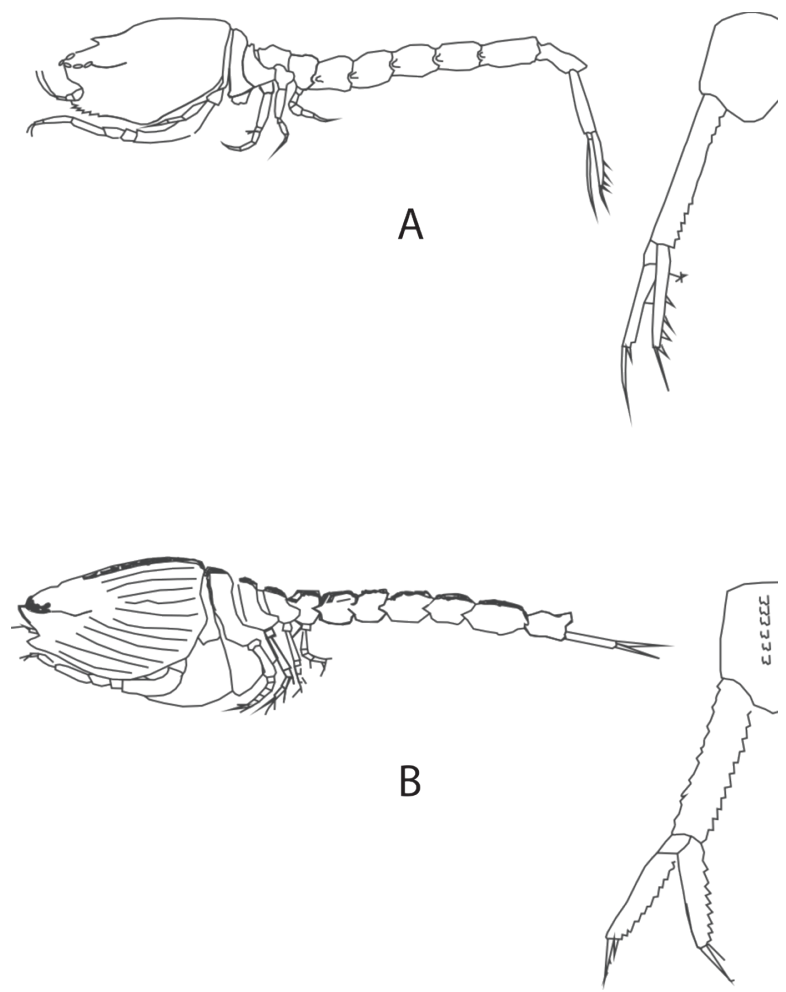

FIGURA 8. A-B. Vista lateral del cuerpo, telson y urópodos. A, Cyclaspis unicornis †. B, C. simonae $q$. 


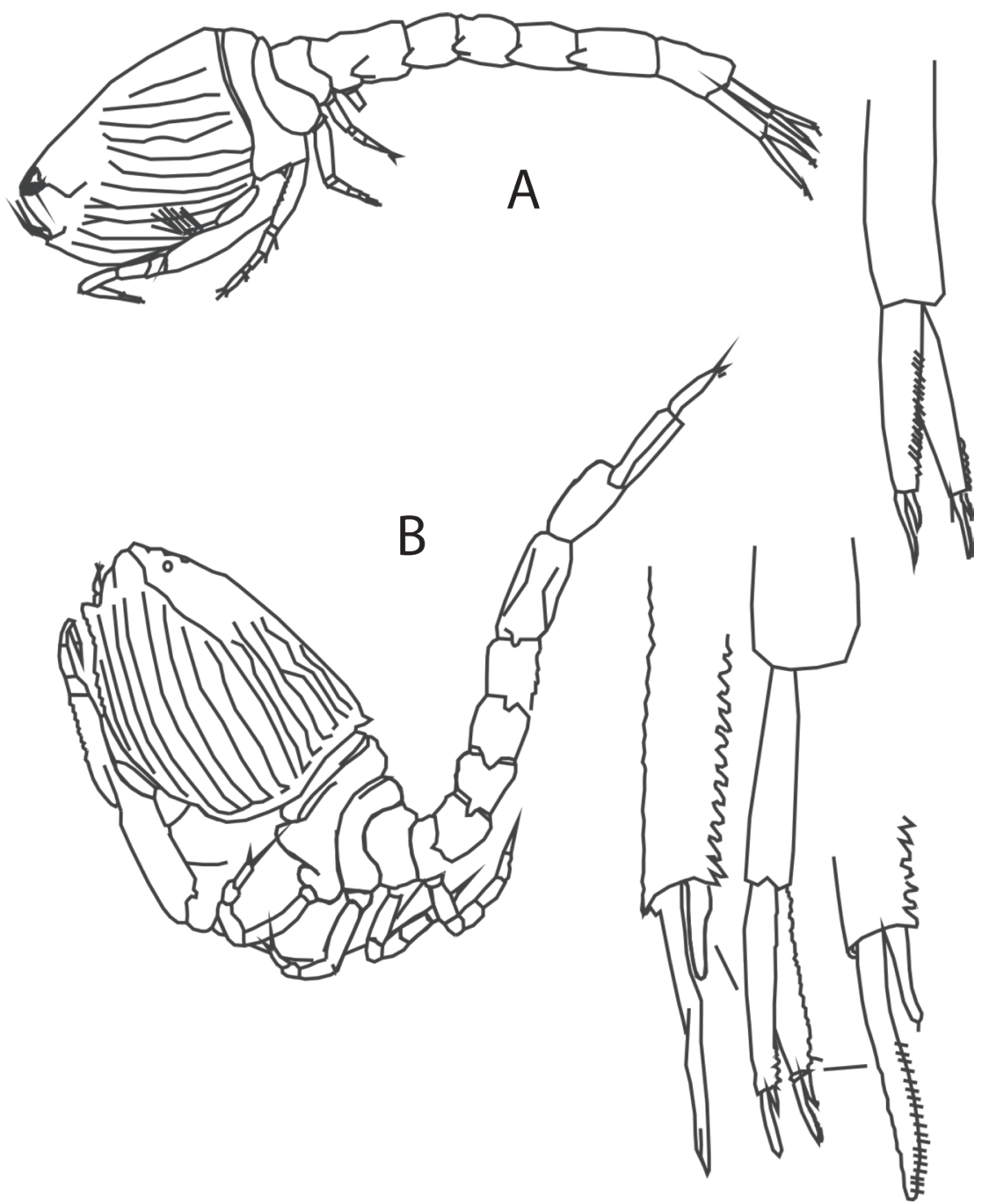

FIGURA 9. A-B. Vista lateral del cuerpo, telson y urópodos. A, Cyclaspis bacescui 우. B, C. iorgui ․

Subfamilia VAUNTHOMPSONIINAE Sars, 1878

Diagnosis. Machos y hembras del mismo tamaño; machos con antenas muy largas que se protegen en canales laterales del carapacho; hembras con exópodos en los pereópodos 1-3 endopodito del urópodo con dos artejos.

\section{Género Vaunthompsonia Bate, 1858}

Diagnosis. Exopoditos en pereiópodos 1-4 en el macho y 1-3 en la hembra; Hembra con al menos tres artejos en la antena. Machos con cinco pares de pleópodos.

Especies citadas para Cuba: V. floridana Băcescu, 1971 (Băcescu y Muradian, 1977); V. minor Zimmer, 1944 (Ortiz y Lalana, 1989). 


\section{CLAVE PARA LA IDENTIFICACIÓN DE LAS HEMBRAS DE LAS ESPECIES CUBANAS}

Hembras con dos hileras longitudinales juntas y paralelas de dientes pequeños en la línea media del carapacho; rostro con 8 lentes que rodean a otro central, todos del mismo diámetro -Vaunthompsonia minor (fig. 10A).

Hembras sin las mencionadas hileras en el rostro; con 6-7 lentes pequeños dorsales que rodean una macha oscura central ----- Vaunthompsonia floridana (fig. 10B).
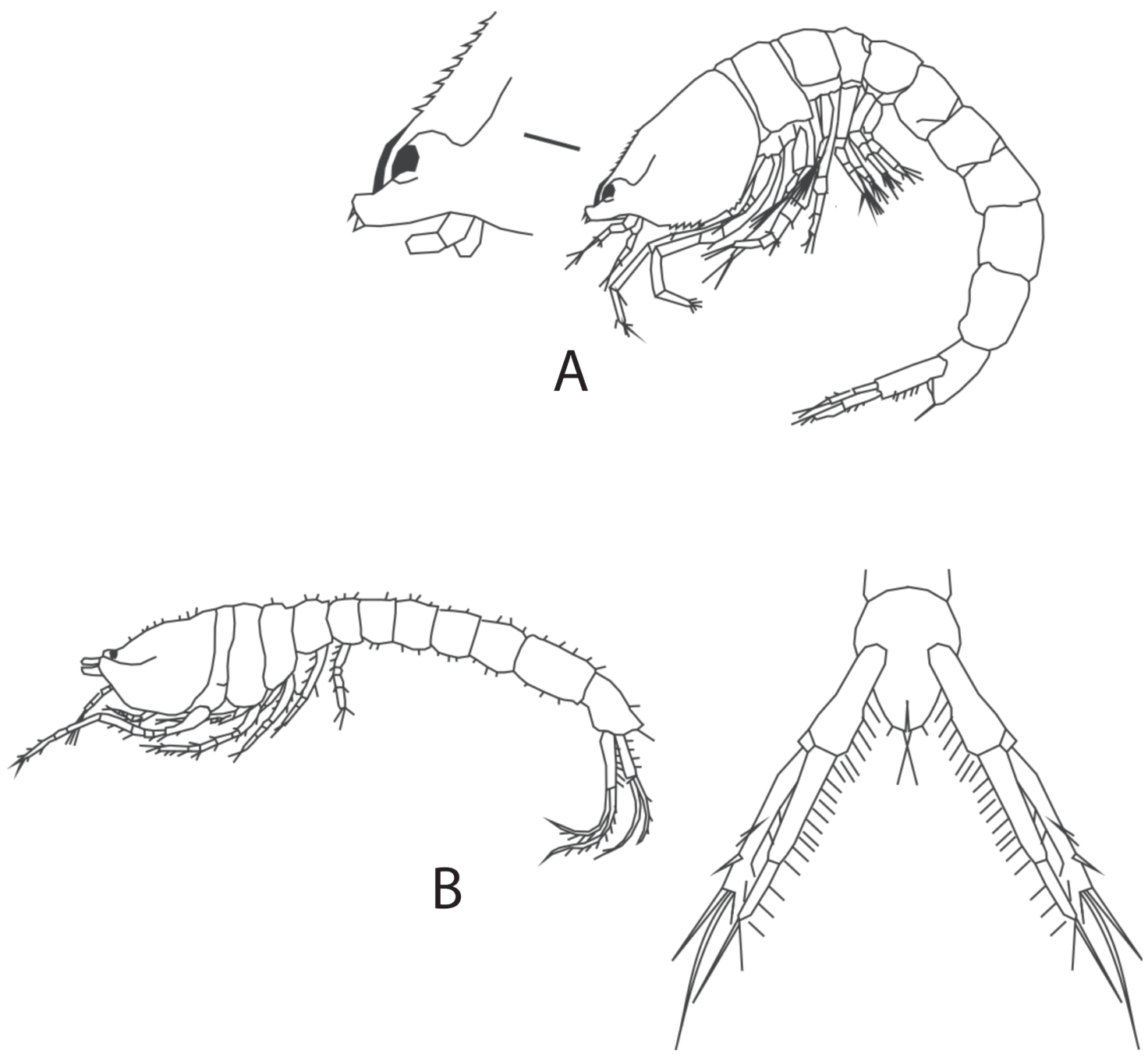

FIGURA 10. A-B. Vista lateral del cuerpo, telson y urópodos. A, Vaunthompsonia minor + . B, V. floridana $q$. 


\section{Familia DIASTYLIDAE Bate, 1856}

La integran a nivel mundial cerca de 18 géneros y más de 300 especies (Heard et al., 2007).

Antenas largas y filamentosas en los machos; cortas en las hembras; poseen un telson libre y alargado, que casi siempre posee dos setas distales. Exópodos presentes en ambos sexos en el tercer par de maxilípedo; en las hembras además, en los tres primeros pares de pereópodos; los machos los llevan en los pereópodos 1-4. Endópodos del urópodo con dos o tres artejos. Telson al menos, aguzado distalmente, generalmente con dos setas cortas dispuestas en su ápice; endópodo con tres artejos.

Género Leptostylis Sars, 1869

Diagnosis. Carapacho en vista lateral con pseudorostro aguzado anteriormente; telson subrectangular, cuarto distal convergiendo bruscamente hacia el ápice; con dos setas distales dispuestas en "V".

Especie citada para Cuba: Leptostylis gutzui Petrescu, 2004 (fig. 11A).

Con las características del género.

Género Oxyurostylis Calman, 1912

Diagnosis. Con un telson inconfundible en forma de estilete doblado ligeramente hacia arriba, sin setas distales.

Especie citada para Cuba: Oxyurostylis cf. smithi (fig. 11B).

Diagnosis. Carapacho con ornamentos en posición variable. Las crestas posteriores a las del pseudorostro separadas entre sí mediante una depresión; telson alargado con extremo distal bífido. (Heard et al., 2007).

\section{Familia LEUCONIDAE Sars, 1878}

Integran esta familia 12 géneros y más de 120 especies, a nivel mundial (Heard et al., 2007).

Se caracterizan por poseer el telson muy reducido y soldado con el sexto segmento pleonal; machos con dos, uno o ningún par de pleópodos; rama interna de los pleópodos sin proceso externo; machos con cinco pares de exópodos (algunos pocos con tres); maxilípedos no branquiales.

Género Leucon Kröyer, 1846. Telson

Diagnosis. Con el margen anterior del pseudorostro sub-agudo, dirigido hacia delante; con abertura sifonal anterior discreta; hembras con una cresta mediodorsal baja, armada de dientes pequeños; machos sin ellos.

Especie citada para Cuba: Leucon sp. (Ortiz y Lalana, 1994 y 1998). 

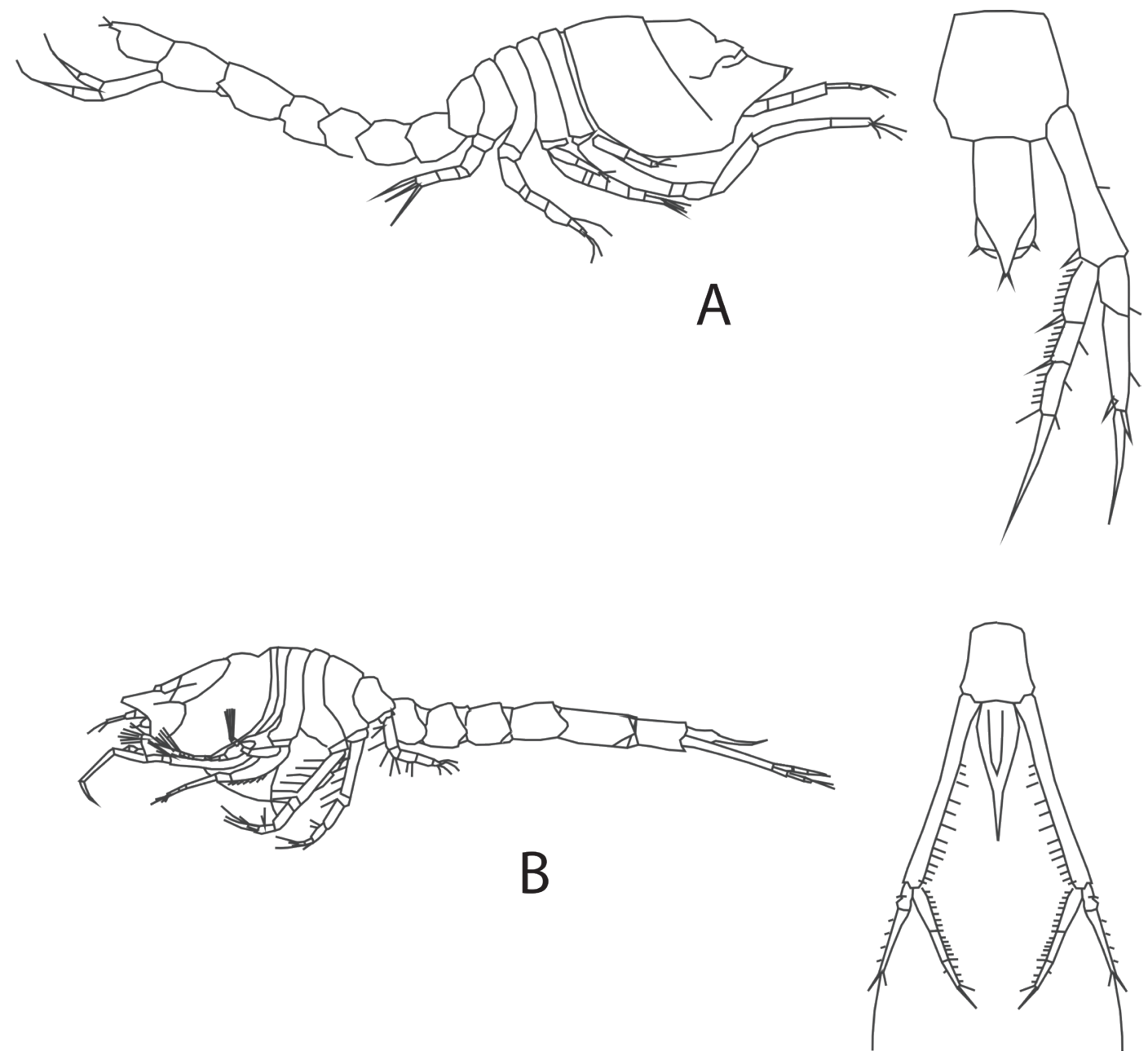

FIGURA 11. Vista lateral del cuerpo, telson y urópodos. A, Leptostylis gutzui 우. B, Oxyurostylis smithi q.

Familia NANNASTACIDAE Bate, 1866

Una de las familias más complejas, con 30 géneros y más de 360 especies en el mundo (Heard et al., 2007). Se caracteriza por presentar el sifón anterior desarrollado; telson muy reducido y soldado al último segmento abdominal, al igual que en las familias Bodotriidae y Leuconidae. No poseen pleópodos; los machos poseen cinco pares de exópodos (raramente cuatro o tres) y tres (casi nunca cuatro o ninguno), en las hembras; mandíbulas con molar estiliforme o cilíndrico y romo distalmente; urópodo con una sola rama. Heard et al. (2007), dividen tentativamente la familia en cuatro grupos: Almyracuma, Campylaspis, Cumella/ Nannatacus y Procampylaspis. Los tres últimos, poseen representantes en las aguas cubanas.

Género Campylaspis Sars, 1865

Diagnosis. Mandíbula con molar estiliforme; maxilípedo dos del tipo "Campylaspis" (fig. 4C); exópodo en maxilípedo tres en ambos sexos; machos con exópodos en los pereópodos 1-4; hembras con exópodos en los pereópodos 1 y 2. 
Especie citada para Cuba: Campylaspis heardi Muradian-Ciamician, 1980 (fig. 12A).

Diagnosis. Carapacho con aspecto reticulado con depresiones cuadrangulares; urópodos con superficie cubierta de finos tubérculos; endópodo con 3-4 setas robustas dirigidas hacia adentro; exópodo con dos artejos; con una seta robusta distal.

Campylaspis sp. (citada por Lalana, Ortiz y Varela, 2005)

Género Cubanocuma Băcescu y Muradian, 1977

Diagnosis. Carapacho en vista lateral subcuadrado; rectangular en vista dorsal; mitad anterodorsal con una cresta casi continua que termina por debajo de los lóbulos del pseudorostro; lóbulo ocular con tres ojos; maxilípedo tres y pereópodos 1.4 con exópodo.

Especie citada para Cuba: Cubanocuma gutzui Băcescu y Muradian, 1977 (fig. 12B).

Con las características del género.

Género Cumella Sars, 1865
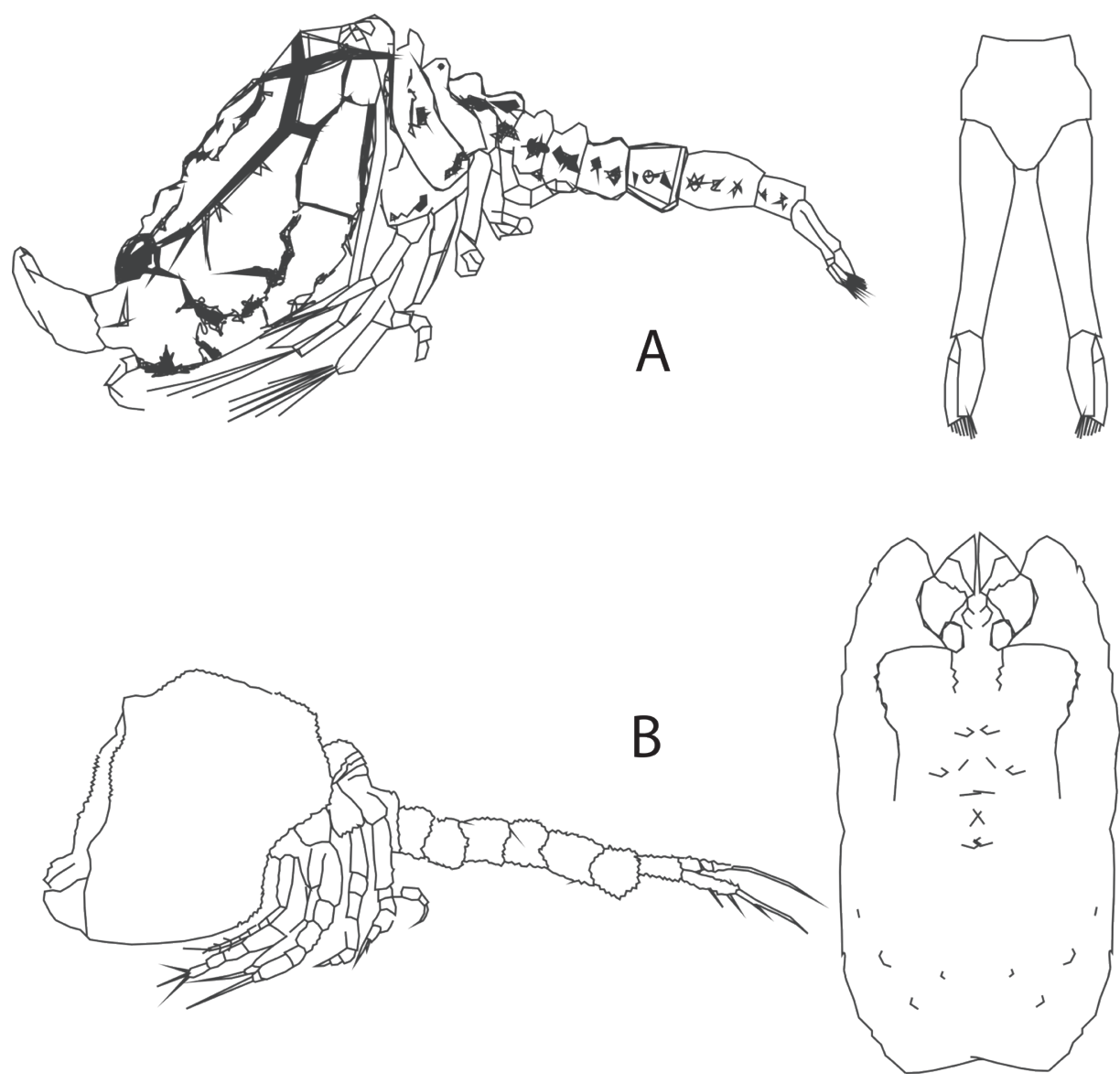

FIGURA 12. Vista lateral del cuerpo, telson y urópodos. A, Campylaspis heardi $\bigcirc$. B, Cubanocuma gutzui $\bigcirc$. 
Diagnosis. Lóbulos oculares formando dos grupos de ommatídeos y lentes; pleonites 1-4 con márgenes laterales con setas largas y espinas; urópodos con setas tan largas como sus ramas; telson soldado al últimos segmento abdominal.

Especies citadas para Cuba: Cumella (Cumella) antipai Petrescu, Iliffe y Sarbu, 1992; Cumella (Cumella) bacescui Petrescu e Iliffe, 1992; Cumella (Cumella) clavicauda Calman, 1911; Cumella (Cumella) coralicola Băcescu, 1971; Cumella (Cumella) garrtyi Băcescu y Muradian, 1977; Cumella (Cumella) gomoiui Băcescu y Muradian, 1977; Cumella (Cumella) ocellata Băcescu, 1992; Cumella (Cumella) serrata Calman, 1911; Cumella (Cumella) vicina Zimmer, 1944; Cumella (Cumewingia) somersi Petruscu y Sterrer, 2001.
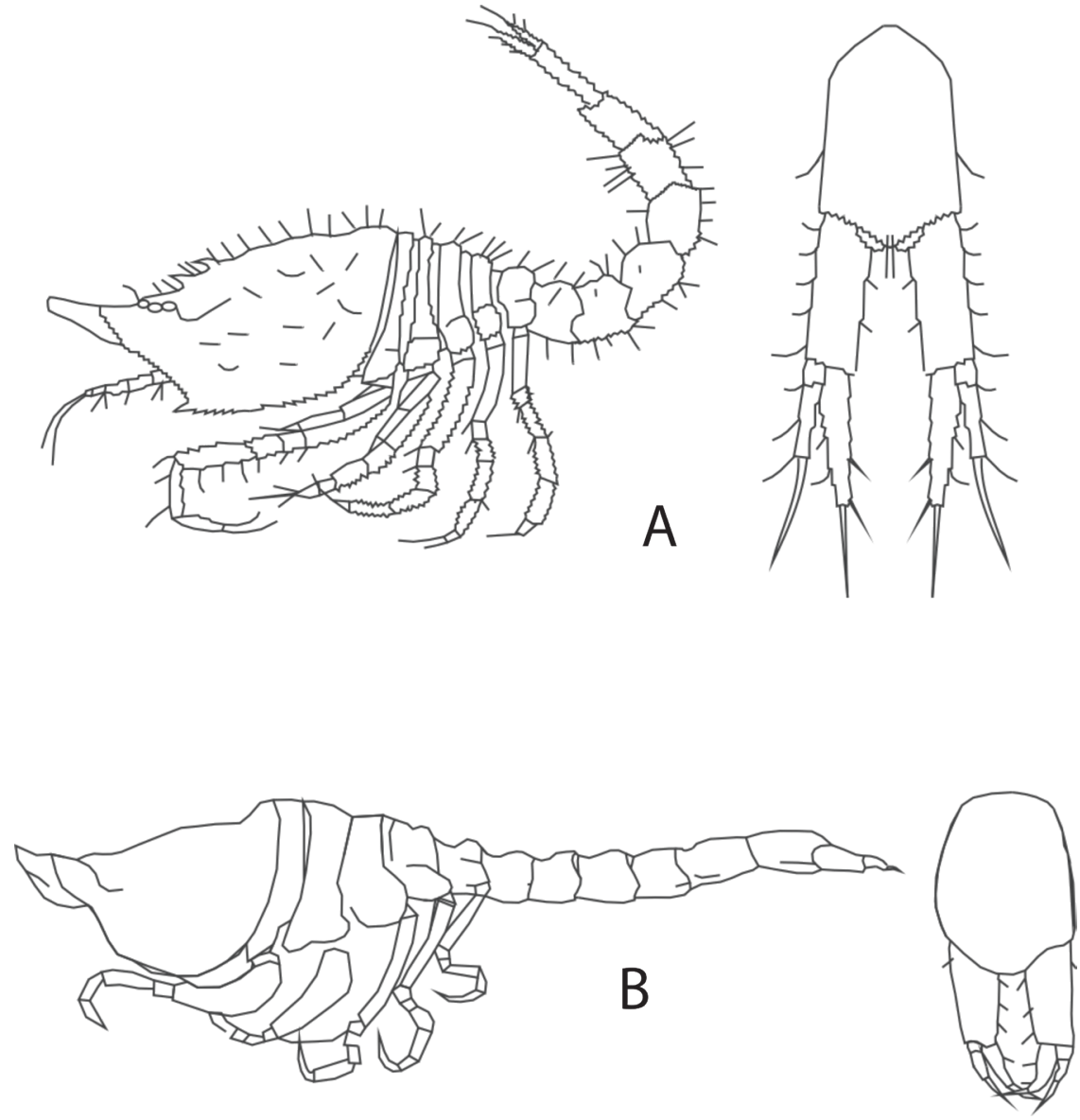

FIGURA 13. Vista lateral del cuerpo, telson y urópodos: A, Cumella somersi $\bigcirc$. B. C. clavicauda $q$. 


\section{CLAVE PARA LA IDENTIFICACIÓN DE LAS HEMBRAS \\ DE LAS ESPECIES CUBANAS DEL GÉNERO CUMELLA}

1A. Carapacho con dos dientes fuertes dirigidos hacia delante en la mitad anterior mediodorsal; margenes ventrales del pseudorostro, resto del carapacho y pereópodos 1-5 finamente aserrados --------------- Cumella somersi (fig.13A).

1B. Carapacho sin los mencionados dientes; pereópodos 1-5 lisos $-2$

2A. Urópodo tan largo o menos que el sexto segmento del abdomen -Cumella clavicauda (fig. 13B).

2B. Urópodo más largo que el sexto segmento del abdomen 3

3A. Con setas dispersas en el carapacho y en el dorso de los segmentos del pleón; telson formando dos lóbulos terminales ----- Cumella coralicola (fig.14A).

3B. Sin setas dispersas en el carapacho y en el dorso de los segmentos del pleón; telson vestigial entero

4A. Con el margen mediodorsal del carapacho uniforme o irregularmente aserrado en toda su extensión, Cumella serrata (fig. 14B).

4B. Con el margen mediodorsal del carapacho liso o crenulado ------------------ 5

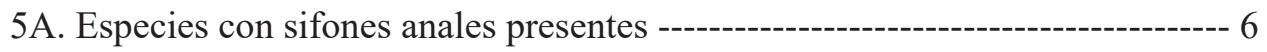

5B. Especies sin sifones anales presentes ------------------------------------------------ 7

6A. Urópodos con el pedúnculo, exo y endopodito con sus márgenes finamente crenulados Cumella bacescui (fig. 15A).

6B. Urópodos con el pedúnculo, exo y endopodito con sus márgenes finamente crenulados Cumella garrityi (fig. 15B).

7A. Carapacho con el margen medio dorsal crenulado (crenulaciones uniformes o irregulares); segmentos de pleón con crestas laterales longitudinales

Cumella vicina (fig. 16A).

7B. Carapacho con el margen mediodorsal liso; segmentos del pleón sin crestas laterales longitudinales 8

8A. Pseudorostro muy aguzado, casi tan largo como el largo del carapacho; con menos de tres ojos, a cada lado; urópodos desnudos totalmente Cumella gomoiui (fig.16B).

8B. Pseudorostro corto, menos de un tercio del largo del carapacho; con más de tres ojos, a cada lado; urópodos con setas robustas en el pedúnculo y ambas ramas del urópodo 
9A. Con los ojos del mismo diámetro; urópodo con el pedúnculo con tres setas robustas en su margen interno; con dos setas robustas en el margen interno del endopodito Cumella ocellata (fig. 17A).

9B. Con los ojos de diferente diámetro; urópodo con el pedúnculo con cinco o seis setas robustas en su margen interno; con cinco setas robustas en el margen interno del endopodito --Cumella antipai (fig. 17B).
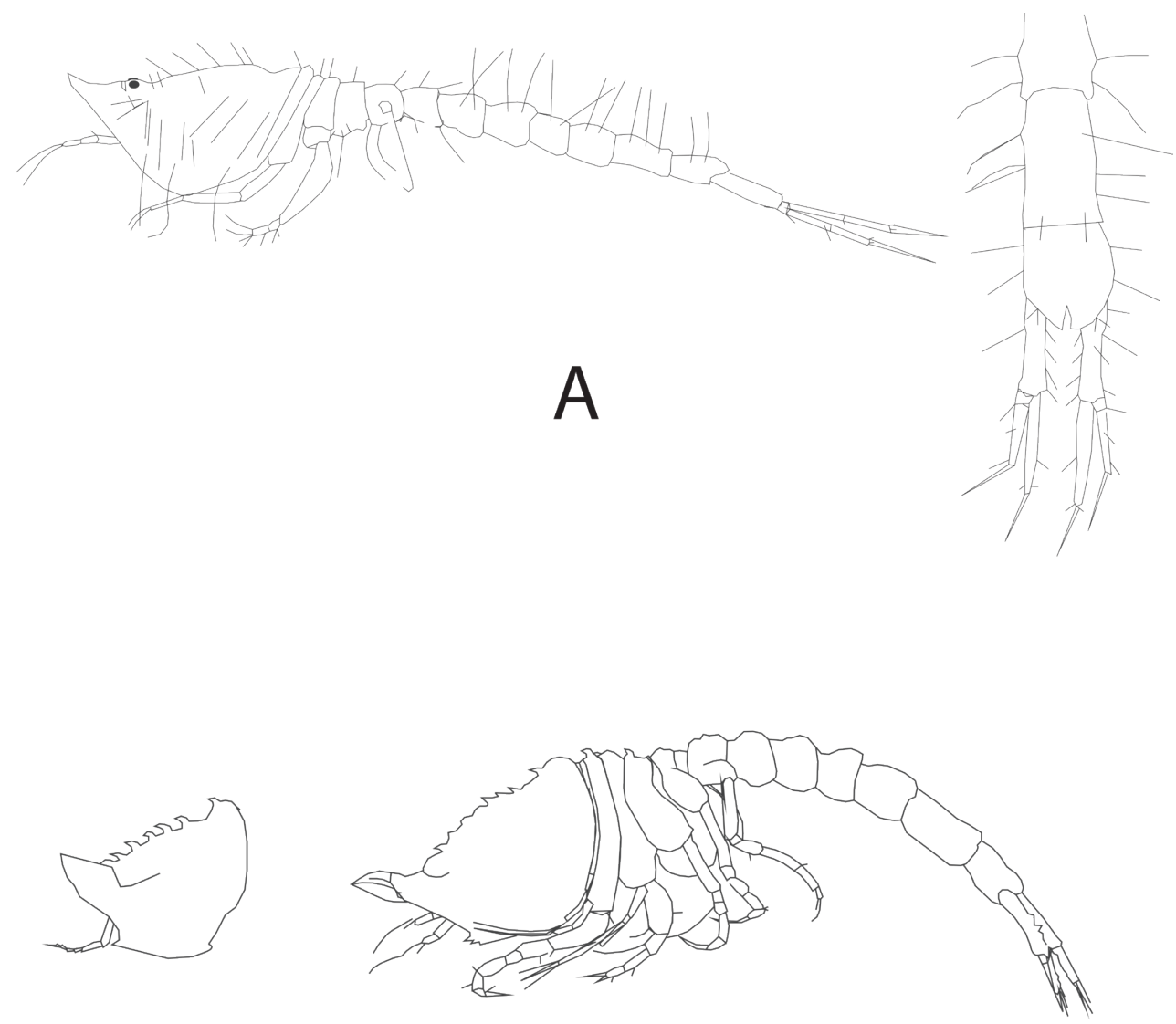

B

FIGURA 14. Vista lateral del cuerpo, telson y urópodos. A, Cumella coralicola ․ B, C. serrata 9. 

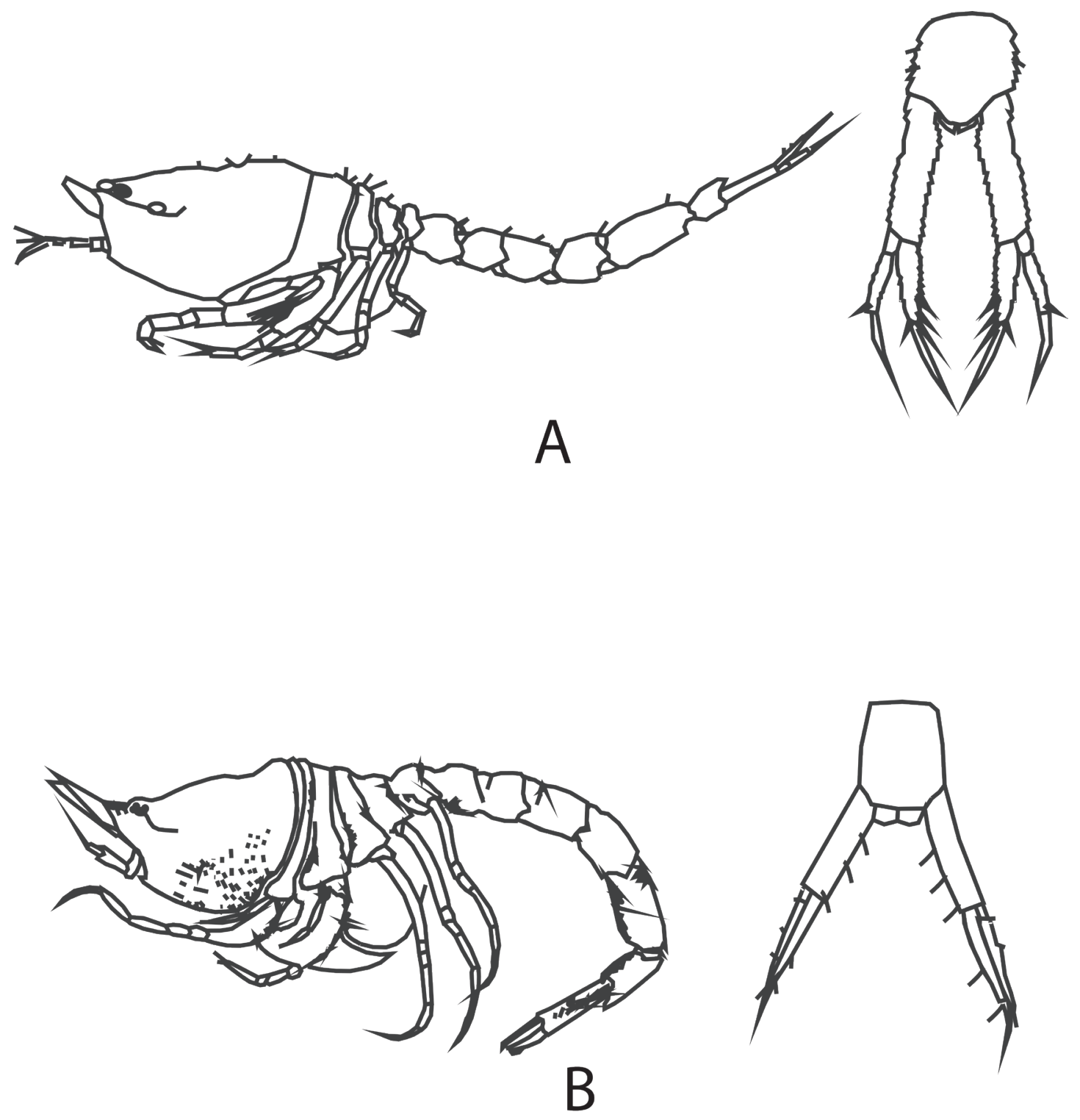

FIGURA 15. Vista lateral del cuerpo, telson y urópodos. A, Cumella bacescui + . B, C. garrityi $^{\circ}$ 

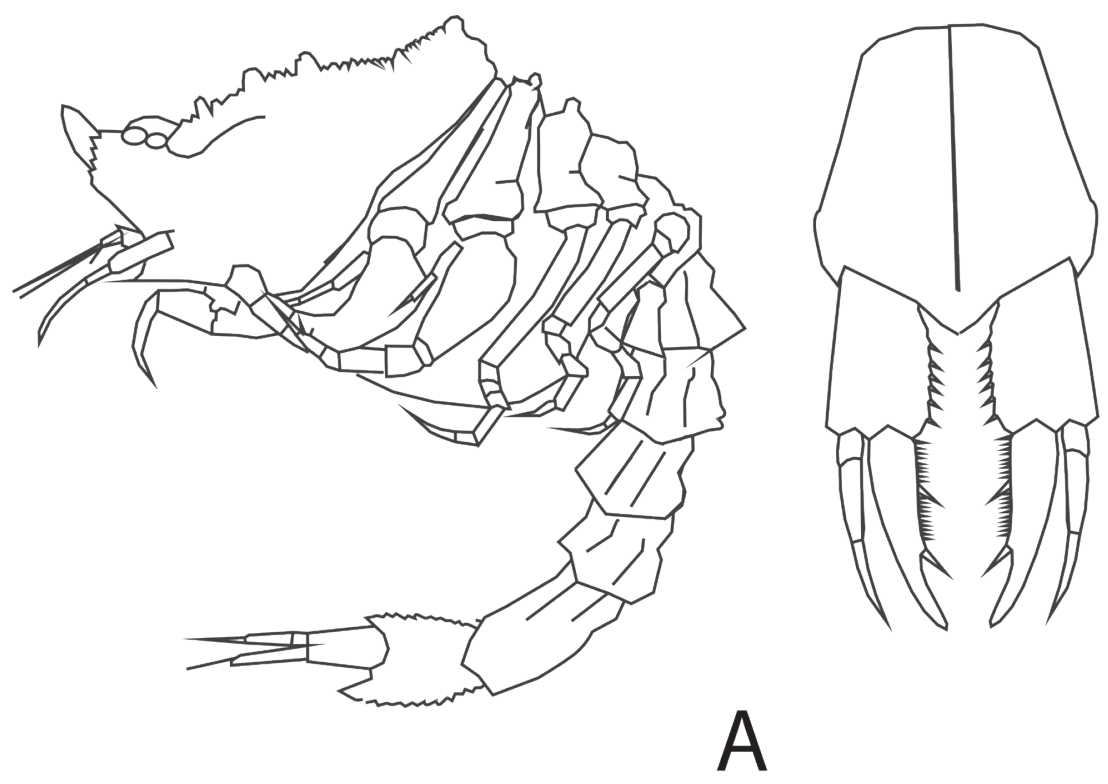

A

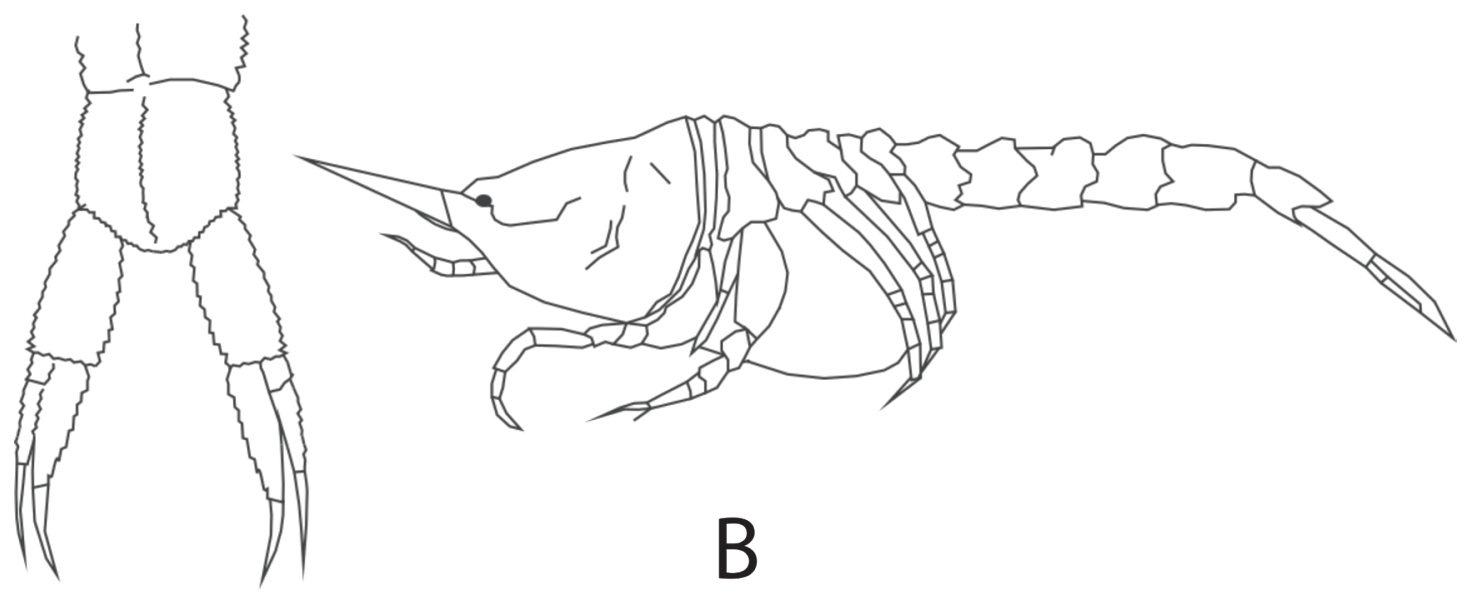

FIGURA 16. Vista lateral del cuerpo, telson y urópodos. A, Cumella vicina + . B. C. gomoiui ${ }_{+}$. 


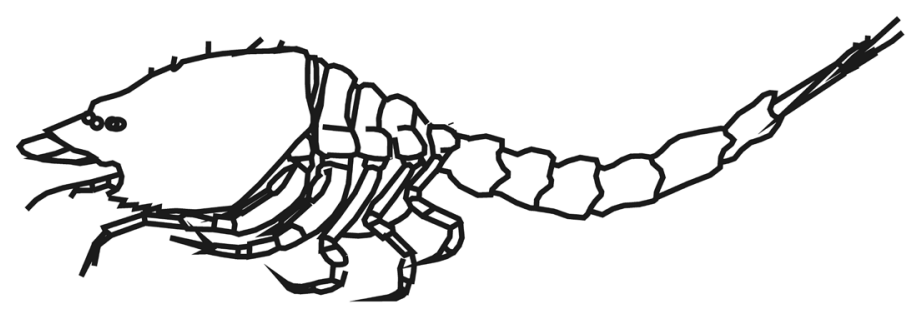

A
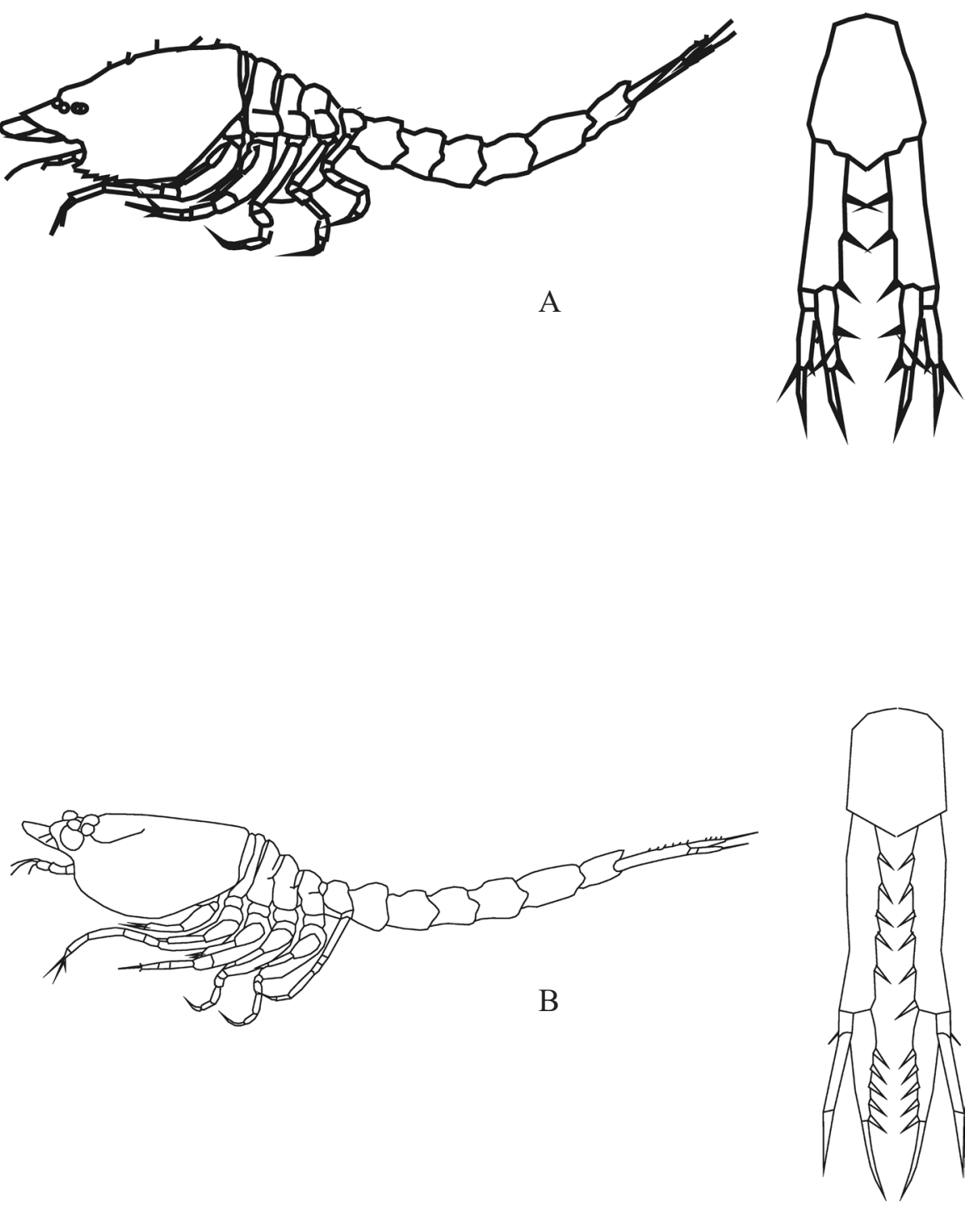

FIGURA 17. Vista lateral del cuerpo, telson y urópodos. A, Cumella ocellata + . B, C. antipai + .

Género Elassocumella Watling, 1991.

Diagnosis. Ojos fusionados; lóbulo medio del carapacho redondeado no llegando a la punta del pseudorostro; urópodo con el exopodito dirigido hacia afuera; endopodito dirigido hacia adentro; pedúnculo más corto que el último segmento pleonal con dos setas robustas dispuestas en su mitad distal; sin sifón rostral; hembras sin exópodos.

Especie citada para Cuba: Elassocumella micruropus (Zimmer, 1861). Figura 18A.

Con las características del género. 
Género Procampylaspis Bonnier, 1896.

Diagnosis. Carapacho generalmente cubierto por una capa de sedimento o detritus; maxilípedo dos con dáctilo digitiforme; pereópodo 1 con el isquio alargado.

Especie citada para Cuba: Procampylaspis sp. (Ortiz et al, 2012).

Género Schizotrema Calman, 1911.

Diagnosis. Carapacho y abdomen con setas o setas robustas en sus pleuras: antena con un tubérculo en el artejo 2 del pedúnculo; pseudorostro con dos aberturas sifonales separadas; mandíbula con molar truncado; machos con exópodos en los pereópodos 1-4; hembras con exópodos en el primer par. La especie cubana suele envolverse en una capa de sedimento aglutinado.

Schizotrema agglutinanta (Băcescu, 1971). Figura 18B.
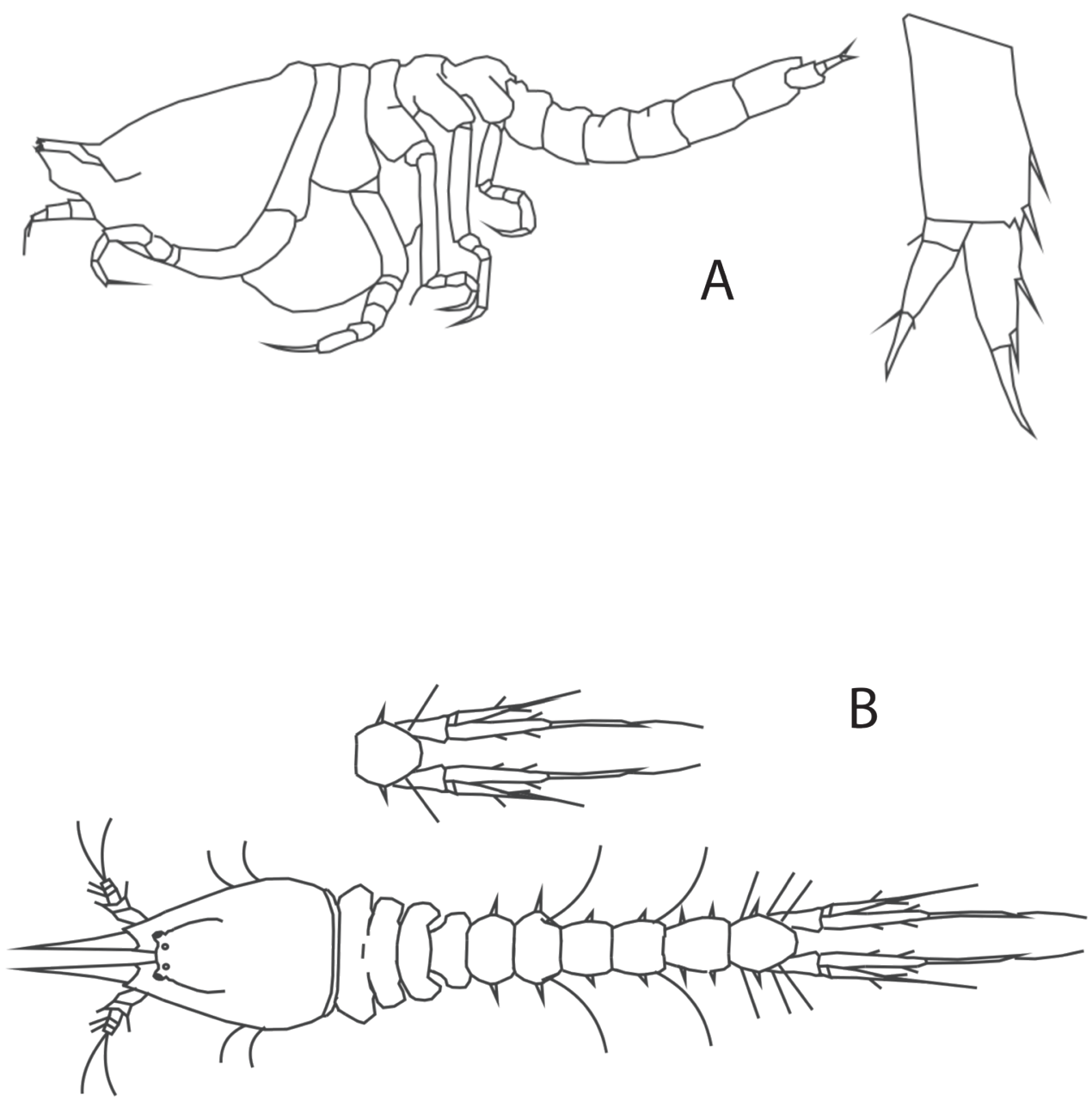

FIGURA 18. Vista lateral del cuerpo, telson y urópodos. A, Elassocumella micruropus 9 . B, Vista dorsal de Schizotrema agglutinanta 9 . 


\section{CONCLUSIONES}

Este trabajo ofrece los aspectos esenciales para lograr la identificación de las especies cubanas del grupo. Debe tenerse en cuenta que los cumáceos cubanos, sin lugar a dudas, aún no están bien estudiados. Una buena parte de las citas existentes provienen del trabajo de Petrescu (2004), que se basó en colectas realizadas en la región suroriental de nuestro archipiélago, hace ya bastante tiempo. De las especies de la región nororiental y de las aguas profundas, no se conoce prácticamente nada. Es por ello, que existen enormes posibilidades de encontrar nuevas especies. Esperemos que las nuevas generaciones de biólogos del país, se encarguen de seguir adelante el estudio de estos interesantes crustáceos peracáridos.

\section{AGRADECIMIENTOS}

Al Dr. Iorgu Petrescu, del Museo Nacional de Historia Natural "Grigore Antipa", de Bucarest, Rumania, por el envío constante de literatura especializada.

\section{LITERATURA CITADA}

Băcescu, M. 1971. New Cumacea from the litoral waters of Florida (Caribbean Sea). Travaux du Museum d'Histoire Naturelle "Grigore Antipa", 11: 5-24.

Băcescu, M. y Z. Muradian. 1977a. Cubanocuma gutzui gen.et.sp.n. (Cumacea, Nannastacidae) from the tropicalwestern Atlantic. Revue Roumaine de Biologie, série de Biologie Animale, 22: 3-9.

Băcescu, M. y Z. Muradian. 1977b. Species of the genus Cumella (Cumacea, Nannastacidae) from the Western Tropical Atlantic. Travaux du Museum d'Histoire naturelle "Grigore Antipa", 28: 89-101.

Băcescu, M. y M. Ortiz. 1984. Contribution to the knowledge of the Mysidacea (Crustacea), of the Cuban insular shelf waters. Travaux du Museum de Historie Naturelle «Grigore Antipa», 26: 15-23.

Calman, W. 1907. On new and rare Crustacea of the order Cumacea from the collection of the Copenhagen Museum, Part 1. The family Bodotridae, Vaunthomsoniidae and Leuconidae. Transactions Zoological Society London, 18 (1): 6-15.

Calman, W. 1912. The Crustacea of the Order Cumacea in the collection of the United State National Museum, Proccedings of the United States Museum of Natural History, 41 (1876): 603-676.

Donath-Henández, F. E. 1988. Cumacea from the Gulf of Mexico and the Caribbean Sea (Crustacea, Peracarida). Descriptions of known species, new records and range extensions. Caribbean Journal of Science, 24 (1-2): 44-51.

Heard, R. W., D. Roccatagliata e I. Petrescu. 2007. An illustrated guide to Cumacea Crustacea: Malacostraca: Peracarida) from Florida coastal and shelf waters to depths of $100 \mathrm{~m}$. State of Florida, Department of environmental protection Tallahassee, 175pp.

Lalana, R., M. Ortiz y C. Varela. 2005. Primera adición a la lista de los crustáceos no decápodos de Cuba. Biología, 19: 50-56. 
Ortiz, M. y R. Lalana. 1988. Lista de especies y bibliografía de los crustáceos de Cuba II. Cirripedia, Phyllocarida, Pancarida, Mysidacea, Tanaidacea y Cumacea. Revista de Investigaciones Marinas, 9 (2): 11-19.

Ortiz, M. y R. Lalana. 1989. Nuevas consignaciones de crustáceos marinos cubanos. Revista de Investigaciones Marinas, 10 (3): 219-221.

Ortiz, M. y R. Lalana. 1994. Lista de especies y bibliografía de los cumáceos del Mediterráneo Americano. Revista de Investigaciones Marinas, 15 (3): 197-200.

Ortiz, M. y R. Lalana. 1998. Lista actualizada de los crustáceos no decápodos de Cuba. Revista de Investigaciones Marinas, 19 (2-3): 92-99.

Ortiz, M. y R. Lalana. 2002. Una nueva especie de cumáceo del género Cyclaspis (Cumacea, Bodotriidae), de aguas cubanas. Avicennia, 15: 23-25.

Ortiz, M. y R. Lalana. 2010. Claves taxonómicas para identificar a crustáceos cubanos (Arthropoda, Crustacea). Cocuyo, 18: 5-28.

Ortiz, M., I. Winfield y C. Varela. 2012. First records of peracarid crustaceans from the Cayo Matias Ocean Blue Hole, SW Cuba, with the description of two new species. Zootaxa, 3505: 53-66.

Petrescu, I. 1996. Cumacea (Crustacea, Cumacea) from Abaco Island (Bahamas). Travaux du Museum d'Histoire Naturelle "Grigore Antipa", 36:157-183.

Petrescu, I. 2002. Crustacea (Crustacea, Peracarida) from Belize. Travaux du Muzeum National d'Histoire Naturelle "Grigore Antipa", 44: 41-203.

Petrescu, I. 2004. New mentions of Cumaceans (Crustacea: Peracarida) in Cuba. Travaux du Muzeum National d'Histoire Naturelle "Grigore Antipa", 47: 89-95.

Sars, G. O. 1871. Beskrivelse af fire vestindiske Cumaceer opdagede af Dr. A. Goës. Öfversigt af Kongliga Vetenskaps-Akademiens Forhandlingar, 28 (6): 803-811.

Varela, C., M. Ortiz y R. Lalana. 2003. Crustáceos (Peracarida y Decapoda), de la costa Sur de la Península de Guanahacabibes, Cuba. Rev. Investigaciones Marinas, 24 (1): 73-76.

Watling, L. 1991. Redescription and revisión of some Nannastacidae (Crustacea, Cumacea). Procceding of the Biological Society of Washington, 104: 751-757.

WoRMS, World Register of Marine Species. Available from http://www.marinespecies.org at VLIZ. Accessed 2017-01-15. doi:10.14284/170. 